\title{
Less than 30 Minutes of Door to Balloon Time can Completely Alter the Clinical Course of the Right Ventricular Myocardial Infarction
}

\author{
Deniz Demirci* and Duygu Ersan Demirci \\ Cardiology Department, University of Health Sciences, Turkey
}

*Corresponding author: Deniz Demirci, Cardiology Department, University of Health Sciences, Antalya Education and Research Hospital, Turkey

\section{ARTICLE INFO \\ Received: 幽 January 31, 2019 \\ Published: 幽 February 13, 2019}

Citation: Deniz Demirci, Duygu Ersan Demirci. Less than 30 Minutes of Door to Balloon Time can Completely Alter the Clinical Course of the Right Ventricular Myocardial Infarction. Biomed J Sci \& Tech Res 14(3)-2019. BJSTR. MS.ID.002559.

Abbreviations: IMI: Inferior Myocardial Infarction; RV: Right Ventricular; RVMI: Right Ventricular Myocardial Infarction; PPCI: Primary Percutaneous Coronary Intervention; RCA: Right Coronary Artery; ECG: Electrocardiography; LVESV: Left Ventricle End-Systolic Volume; LVEDV: Left Ventricle End-Diastolic Volume; LVEF: LV Ejection Fraction; RVDD: RV End-Diastolic Diameter; RVFAC: RV Fractional Area Change; TAPSE: Tricuspid Annular Plane Systolic Excursion; STEMI: ST Elevation Myocardial Infarction
ABSTRACT

Introduction: The purpose of the current study was to comparison of the major and minor complications in patients with inferior myocardial infarction (IMI) with or without RVMI after treated with early successful primary percutaneous coronary intervention.

Methods: Consecutive patients admitted with IMI due to proximal RCA occlusion and treated with successful primary PCI were included the study. Patients divided into two groups as IMI with and without RVMI. RVMI was diagnosed in the presence of ST segment elevation in the V4R derivation. In addition, transthoracic echocardiography is performed within 24 hours of admission. All patients were followed prospectively and the occurrence of adverse events was noted. The mean follow-up period was 10 months after discharge.

Results: The study population consisted of 46 consecutive patients admitted with IMI treated with primary PCI. 16 patients were IMI complicated by RVMI. The average door-to-balloon (D2B) times at our study population was less than thirty minutes (mean $23.1 \pm 6,6$ minutes). Patients with RVMI had significantly lower TAPSE $(1.39 \pm 0.21 \mathrm{~cm}$ versus $2.35 \pm 0.46 \mathrm{~cm}, \mathrm{P}<0.001)$ and $\operatorname{RV~FAC~}(28.15 \pm 5.87$ versus $44.79 \pm 10.77 \mathrm{p}<0,001)$ and significantly higher RVDD $(4,17 \pm 0,41$ versus $3,06 \pm 0.45, p<0,001)$. No mortality or major complications were observed during the coronary intensive care and hospital stay. Minor complication was observed in one patient with RVMI, no statistically significant difference was found in this value. No statistically significant difference was found in length of hospital stay. A significant correlation was found between the peak CK-MB values between, both length of coronary intensive care and length hospital stay of patients with RVMI.

Conclusion: In patients with early-successful reperfusion therapy and door-toballoon time is less than 30 minutes, there is no mortality and morbidity difference between IMI with or without complicated RV MI.

\section{What is Already Known?}

Inferior MI complicated with right ventricular MI has a higher mortality rate than isolated inferior MI. Early revascularization is the key to reduce mortality rates in this high-risk subset.

\section{What this Study Adds?}

The study showed that, there is no difference in mortality and morbidity between patients with pure IMI and patients complicated by RVMI, when early and successful reperfusion therapy was performed with a D2B time of less than 30 minutes. According to our results, the main target in the therapy of RVMI should be decreased total ischemic time and increased post-PCI TIMI flows.

\section{Introduction}

There is limited data on the association between right ventricular (RV) function and adverse events after acute right ventricular 
myocardial infarction (RVMI) [1]. Most of the information on RVMI is from the pre-mechanical revascularization era. RVMI increases the risk of hemodynamic instability, atrioventricular conduction block and increases in-hospital mortality in patients with inferior myocardial infarction (IMI) [2,3]. Significant improvement in the prognosis of RVMI was achieved after reperfusion treatments. However, studies have demonstrated that RVMI is still associated with significant morbidity and mortality, even in the mechanical reperfusion era [4]. A shorter time taken to reperfusion of the occluded vessel contributes to the recovery of RV function. Early revascularization plays an important role in the recovery of RV function; conversely, late revascularization is associated with higher RV dysfunction and complications [5,6]. The purpose of the current study was to investigate the complication rates in patients with IMI with and without RVMI who were treated with early primary percutaneous coronary intervention (PPCI) successfully.

\section{Methods}

\section{Patient Selection and Study Protocol}

Consecutive patients, admitted with IMI due to proximal right coronary artery (RCA) occlusion and treated with successful PPCI, were included to the study. There was no critical stenosis in the other vessels

Successful PPCI was defined with three criteria:

a) TIMI 3 coronary flow (complete perfusion) after PPCI.

b) Resolution of ST segment elevation $>70 \%$

c) Complete relief of chest pain.

If any of these three criteria was absent, the patient was excluded from the study. The population of this study was specified as patients who have had chest pain within 6 hours. The patients with onset of symptoms over 6 hours were excluded. IMI was diagnosed as the presence of ST segment elevation of at least $0.1 \mathrm{mV}$ in 2 or more inferior leads on electrocardiography (ECG). RVMI was diagnosed as the presence of ST segment elevation in the V4R lead on ECG. All patients were treated according to the institutional acute myocardial infarction protocol, which is driven by the most recent guidelines. In addition, 2D echocardiography was performed to all patients within 24 hours of admission. LV and RV function were determined by echocardiography. All patients were followed prospectively for the occurrence of adverse events.

Data recorded for all patients included demographics, ECG findings, a detailed timeline including time of onset of chest pain, time of arrival at hospital ("door" time) and time of first balloon inflation of the primary angioplasty procedure ("balloon" time). The time interval between the patient's first arrival at hospital and first balloon inflation was called as door-to-balloon (D2B) time. TIMI risk scores were calculated from the initial clinic history, electrocardiograms, laboratory values collected on admission. After discharge, the patients were evaluated by telephone calls or by hospital checkup programs. The mean follow-up period was 10 months after discharge. The study protocol was approved by the Local Ethics Committee.

\section{Echocardiography}

Images were obtained in the left lateral decubitus position using a commercially available system (Vivid 3 pro General Electric-Vingmed, Horton, Norway). Data acquisition was performed at a depth of $16 \mathrm{~cm}$ in the parasternal and apical views using a 3.5MHz transducer. During breath-hold 2D images were obtained and 3 consecutive beats were saved in cineloop format. The reference limits of all echocardiographic parameters were defined according to American Society of Echocardiography Guidelines. The left ventricle end-systolic volume (LVESV), left ventricle end-diastolic volume (LVEDV) were assessed and LV ejection fraction (LVEF) was calculated by using the biplane Simpson method [7].

\section{RV Function Analysis}

$\mathrm{RV}$ end-diastolic diameter (RVDD) was measured in the apical 4- chamber view. RV fractional area change (RVFAC) was analyzed by tracing the RV end-diastolic area (RVDA) and RV end-systolic area (RVSA) in the apical 4-chamber view using the formula (RVDA-RVSA)/ RVDA x 100. Tricuspid annular plane systolic excursion (TAPSE) was measured in the RV free wall. In the apical 4-chamber view, the M-mode cursor was placed through the tricuspid annulus in such a way that the annulus moved along the M-mode cursor and the total displacement of the RV base from end-diastole to end-systole was measured [8].

\section{Statistical Analysis}

Continuous data were presented as mean standard deviation and categorical data were presented as frequencies and percentages. Differences in characteristics between patient groups were evaluated using the unpaired Student $t$ test and chi-square test. Pearson's chi-square test was used for non-qualitative data when the data were examined (Fisher's exact Chi-square test was used when the number of value ( $\mathrm{n}$ ) in boxes was below 5). Mann-Whitney $U$ test was used for data not showing normal distribution and Student $\mathrm{T}$ test was used for data with normal distribution. The $95 \%$ confidence interval was determined and differences were considered significant at $P<0.05$.

\section{Results}

The study population consisted of 46 consecutive patients, admitted with IMI and treated with PPCI. 16 patients had IMI complicated by RVMI. Table 1 summarizes the clinical characteristics of the patient population. Mean age was 56 years, and most patients were men (n: 39; $85 \%$ ). The patients presented a mean of $146 \pm$ 117 minutes after the onset of symptoms. The average D2B time at our study population was less than 30 minutes (mean $23.1 \pm 6.6$ minutes). Patients with RVMI had significantly lower TAPSE (1.39 
$\pm 0.21 \mathrm{~cm}$ vs $2.35 \pm 0.46 \mathrm{~cm}, \mathrm{P}<0.001)$ and RVFAC $(28.15 \pm 5.87 \mathrm{vs}$ $44.79 \pm 10.77 \mathrm{p}<0,001)$ and significantly higher RVDD (4.17 cm \pm 0.41 vs $3.06 \pm 0.45, p<0.001$ ). No difference was observed in LVEF between the patients with and without RVMI (45.38 $\pm 5.19 \%$ vs $44.16 \pm 6.20 \%, \mathrm{P}>0.05$ ) (Table 2 ). No mortality or major compli- cation was observed during the intensive coronary care unit (ICCU) and hospital stay. A minor complication was observed in one patient with RVMI, this was not statistically significant ( $p>0.05$ ). Atrial fibrillation was detected in one patient in IMI group and this was not statistically significant too $(\mathrm{P}>0.05)$ (Table 3$)$.

Table 1: Baseline Clinical Characteristics.

\begin{tabular}{|c|c|c|c|}
\hline & \multicolumn{2}{|c|}{ Baseline Clinical Characteristics } & p \\
\hline & IMI (n:29) & RVMI (n:17) & $>0,05$ \\
\hline Age (years) & $57 \pm 11$ & $55 \pm 12$ & $>0,05$ \\
\hline Male gender n (\%) & $24(\% 83)$ & $15(\% 88)$ & $>0,05$ \\
\hline HT n (\%) & $13(\% 45)$ & $8(\% 47)$ & $>0,05$ \\
\hline DM n (\%) & $12(\% 42)$ & $8(\% 47)$ & $>0,05$ \\
\hline Family History n (\%) & $9(\% 31)$ & $4(\% 23,5)$ & $>0,05$ \\
\hline Smoking n (\%) & $15(\% 52)$ & $8(47,1)$ & $>0,05$ \\
\hline Symptom onset - door time (minute \pm SD) & $145 \pm 116$ & $148 \pm 118$ & $>0,05$ \\
\hline Door- to-balloon time (minute \pm SD) & $22 \pm 7$ & $25 \pm 6,1$ & $>0,05$ \\
\hline TIMI risk score & $2,83 \pm 1,00$ & $2,82 \pm 0,95$ & \\
\hline
\end{tabular}

DM: Diabetes Mellitus, HT: Hypertension, IMI: Inferior myocardial infarction, RVMI: Right ventricular myocardial infarction SD: Standard deviation, TIMI: Thrombolysis in Myocardial Infarction

Table 2: Comparison of Echocardiographic Findings.

\begin{tabular}{|c|c|c|c|}
\hline & Comparison of Echocardiographic Findings & p \\
\hline & IMI & RVMI & $>0,05$ \\
\hline LVEF (\%) & $44,16 \pm 6,20$ & $45,38 \pm 5,19$ & $<0,001$ \\
\hline TAPSE (cm) & $2,35 \pm 0,46$ & $1,39 \pm 0,21$ & $<0,01$ \\
\hline RVDD (cm) & $3,06 \pm 0,45$ & $4,17 \pm 0,41$ & $<0,01$ \\
\hline
\end{tabular}

IMI: Inferior myocardial infarction, LVEF: left ventricular ejection fraction, RVFAC: Right ventricular fractional area change, RVMI: Right ventricular myocardial infarction, TAPSE: Tricuspid annular plane systolic excursion

Table 3: Comparison of Major and Minor Complication Rates

\begin{tabular}{|c|c|c|c|}
\hline \multicolumn{4}{|c|}{ Comparison of Major and Minor Complication Rates } \\
\hline Major Complications & IMI & RVMI & $\mathbf{p}$ \\
\hline Death & 0 & 0 & \\
\hline Cardiogenic Shock & 0 & 0 & \\
\hline Ventricular fibrillation & 0 & 0 & \\
\hline Complete AV block & 0 & 0 & \\
\hline Other rhythm disorders that require PM implantation & 0 & 0 & \\
\hline \multicolumn{4}{|l|}{ Minor Complications } \\
\hline Symptomatic other AV blocks & 0 & 0 & \\
\hline Symptomatic sinus bradycardia & 0 & 0 & \\
\hline Intra-aortic balloon pump requirement & 0 & 0 & \\
\hline Atrial Fibrillation & 1 & & $>0,05$ \\
\hline Symptomatic hypotension* & 0 & 1 & $>0,05$ \\
\hline
\end{tabular}

IMI: Inferior myocardial infarction, PM: Pace Maker, RVMI: Right ventricular myocardial infarction

*Inotropic treatment requirement symptomatic hypotension. 
Length of stay in ICCU was slightly higher in RVMI group but it was not statistically significant ( $p>0.05$ ) (Table 4). There was no statistically significant difference between two groups with respect to peak CK-MB levels ( $p>0.05$ ). When correlation analysis was performed between peak CK-MB values and length of hospital stay, no significant correlation was found between the length of ICCU / hospital stay and peak CK-MB values in patients with IMI ( $p>0.05$ ). However, a significant positive correlation was found between the peak CK-MB values and both length of ICCU and hospital stay in patients with RVMI. (Hospital stay r: 0.668, p<0.001; ICCU stay r: $0.803, p<0.001$ ). After an average of 10 months follow up of patients, no new infarction or mortality was observed in both groups.

Table 4: Comparison of the length of stay in hospital and coronary care unit.

\begin{tabular}{|c|c|c|c|}
\hline & \multicolumn{3}{|c|}{ Comparison of the Length of Stay in Hospital and Coronary Care Unit } \\
\hline & IMI & RVMI & P value \\
\hline In hospital & $5,69 \pm 2,67$ & $3,00 \pm 3,64$ & $>0,05$ \\
\hline In coronary care unit & $2,51 \pm 0,94$ & $3,24 \pm 2,31$ \\
\hline
\end{tabular}

\section{Discussion}

Our study showed that IMI complicated with RVMI did not increase mortality and morbidity when early and successful reperfusion was performed. Recently many clinical trials showed that, rapid administration of intravenous thrombolytic therapy and increasing use of primary angioplasty reduce mortality and contribute to the shorter hospital stays $[9,10]$. Although the benefits of these treatments have been demonstrated, over $25 \%$ of patients presenting within the first $12 \mathrm{~h}$ of symptom onset with ST segment elevation or left bundle branch block fail to receive a reperfusion intervention. It is clear that outcomes for ST elevation myocardial infarction (STEMI) patients are optimized when D2B times are less than 90 minutes $[11,12]$. Multiple studies also demonstrated that mortality rates improve by achieving decreased D2B times [13]. A review of a large cardiovascular registry $(10,965$ patients) found that the median D2B time was 96 minutes and only $44 \%$ of the patients were meeting the $<90$-minute national benchmark goal [14]. Cannon et al. showed that mortality increased by $41 \%$ for STEMI patients with D2B times over 120 minutes [10].

In our study, the mean D2B time was less than half an hour. Furthermore, the median time from onset of chest pain to hospital arrival was less than 2.6 hours and the median time from onset of chest pain to PPCI was less than 3 hours. The low rate of mortality and morbidity in our study can be explained by early reperfusion therapy. The risk of major complications and in-hospital death is about $31 \%$ in IMI patients with RVMI compared to about 5\% in IMI without RVMI [2]. The analysis of the SHOCK registry data showed that the patients with predominant RV shock have a high mortality rate despite the younger age, lower rate of anterior MI, and higher prevalence of single-vessel coronary disease. Early reperfusion (PPCI or fibrinolytic therapy) can preserve both right and left ventricular function and also reduce morbidity and mortality [15-17]. Schuler et al. indicated that in most patients with proximal occlusion of the RCA, early reperfusion with thrombolytic therapy contributes to improvement of initially depressed RV function; often, recovery of RV myocardial function is not associated with the changes in LVEF. In contrast, in patients with persistent occlusion, irreversible cardiogenic shock and early death may occur due to RV infarction, despite adequate LV performance [16].

Bowers et al. demonstrated that, complete reperfusion of the RCA by PPCI, results in the dramatic recovery of RV function and an excellent clinical outcome, in patients with RVMI. In contrast, unsuccessful or late reperfusion results in impaired recovery of RV function, persistent hemodynamic compromise, and a high mortality rate [15]. Our study population consisted of patients who underwent early and successful reperfusion treatment. And our results showed that there is no increase in the rate of major complications and in-hospital death in patients with IMI complicated with RVMI compared to IMI without RVMI, when early successful reperfusion therapy was performed. Right-sided ST-segment elevation, particularly in lead V4R, indicates acute RV injury and correlates closely with the proximal RCA occlusion $[18,19]$. In one report of 200 consecutive patients with acute IMI, the sensitivity and specificity of ST-segment elevation in V4R was shown as $88 \%$ and 78 $\%$ (respectively) for concurrent RVMI, by using findings from the results of autopsy, cardiac catheterization, radionuclide imaging, or hemodynamic monitoring as the "gold standard" [2]. Zehender et al. demonstrated that the presence of ST-segment elevation in lead V4R predicts development of major complications and in-hospital mortality strongly and independently [2].

The presence of ST-segment elevation in lead V4R was one of the inclusion criteria for RVMI group in our study. We showed that ST- segment elevation in lead V4R was not an independent predictor of major complications and in-hospital mortality after early successful reperfusion treatment. Anavekar et al. and Zornoff et al. showed that RV function was weakly correlated with LV function and demonstrated that RV function quantified with RVFAC was independently associated with an increased risk of mortality and heart failure [20,21]. Antoni at al. showed that RVFAC and RV strain were independent predictors of the occurrence of the composite end points (all-cause mortality, reinfarction, and hospitalization for HF) [1]. Samad et al. showed that TAPSE predicted mortality independently, after adjustment for LVEF and age [22]. We found that RVFAC and TAPSE values were significantly lower and RV diameter 
was significantly higher in RVMI group. However, we showed that they were not predictors of mortality and morbidity when early and successful reperfusion performed. Gonzales et al. demonstrated that STEMI patients with TIMI risk classification 0-1, 2-3, and $\geq 4$ has in-hospital mortality of $7 \%, 13 \%$ and $26 \%$, respectively [23].

In our study, the mean TIMI risk score of the patients was $2.83 \pm 1.0$. However, after early reperfusion treatment there was no mortality in our study. We showed that clinical outcomes could be changed by early reperfusion therapy in patients with RVMI. We found a significant correlation between peak CK-MB level and length of ICCU/ hospital stay in RVMI patients. In fact, there was no statistically significant difference with respect to peak CK-MB level between patients with and without RVMI. Furthermore, the peak CK-MB level was not associated with the development of complications and the length of hospital stay in patients without RVMI. However, the length of hospital stay was prolonged in patients complicated with RVMI who have higher peak CK-MB levels. This was substantially related to increased length of ICCU stay. Increased length of ICCU stay was probably due to the clinician's concern about RVMI-related complications. Our study showed that the increase of peak CK-MB level was not associated with the development of complications when early and successful reperfusion was performed.

\section{Limitations}

The main limitation of our study was the small number of patients. Our results should be tested with more patients in largescale studies. The absence of the patients who have longer door to balloon times may be accepted as a limitation of the study, but the mean door to balloon time is short in our center.

\section{Conclusion}

We conclude that, there is no difference in mortality and morbidity between patients with pure IMI and patients complicated by RVMI, when early and successful reperfusion therapy was performed with a D2B time of less than 30 minutes.

\section{References}

1. Antoni ML, Scherptong RW, Atary JZ, Boersma E, Holman ER, et al. (2010) Prognostic value of right ventricular function in patients after acute myocardial infarction treated with primary percutaneous coronary intervention. Circ Cardiovasc Imaging 3(3): 264-271.

2. Zehender M, Kasper W, Kauder E, Schonthaler M, Geibel A, et al. (1993) Right ventricular infarction as an independent predictor of prognosis after acute inferior myocardial infarction. N Engl J Med 328(14): 981988.

3. Rodrigues EA, Dewhurst NG, Smart LM, Hannan WJ, Muir AL (1986) Diagnosis and prognosis of right ventricular infarction. Br Heart J 56(1): 19-26.

4. Inohara T, Kohsaka S, Fukuda K, Menon V (2013) The challenges in the management of right ventricular infarction. Eur Heart J Acute Cardiovasc Care 2(3): 226-234.

5. Kidawa M, Kasprzak JD, Wierzchowski T, Krzeminska Pakula M (2010) Right ventricular function suffers from reperfusion delay: tissue Doppler study. Clin Cardiol 33(3): E43-48.
6. Laster SB, Ohnishi Y, Saffitz JE, Goldstein JA (1994) Effects of reperfusion on ischemic right ventricular dysfunction. Disparate mechanisms of benefit related to duration of ischemia. Circulation 90(3): 1398-1409.

7. Lang RM, Bierig M, Devereux RB, Flachskampf FA, Foster E, et al. (2005) Recommendations for chamber quantification: a report from the American Society of Echocardiography's Guidelines and Standards Committee and the Chamber Quantification Writing Group, developed in conjunction with the European Association of Echocardiography, a branch of the European Society of Cardiology. J Am Soc Echocardiogr 18(12): 1440-1463.

8. Rudski LG, Lai WW, Afilalo J, Hua L, Handschumacher MD, Chandrasekaran K, et al. (2010) Guidelines for the echocardiographic assessment of the right heart in adults: a report from the American Society of Echocardiography endorsed by the European Association of Echocardiography, a registered branch of the European Society of Cardiology, and the Canadian Society of Echocardiography. J Am Soc Echocardiogr 23(7): 685-713.

9. Rogers WJ, Canto JG, Lambrew CT, Tiefenbrunn AJ, Kinkaid B, et al. (2000) Temporal trends in the treatment of over 1.5 million patients with myocardial infarction in the US from 1990 through 1999: the National Registry of Myocardial Infarction 1, 2 and 3. J Am Coll Cardiol 36(7): 2056-2063.

10. Cannon CP, Gibson CM, Lambrew CT, Shoultz DA, Levy D, et al. (2000) Relationship of symptom-onset-to-balloon time and door-to-balloon time with mortality in patients undergoing angioplasty for acute myocardial infarction. JAMA 283(22): 2941-2947.

11. Ryan TJ, Antman EM, Brooks NH, Califf RM, Hillis LD, et al. (1999) 1999 update: ACC/AHA Guidelines for the Management of Patients with Acute Myocardial Infarction: Executive Summary and Recommendations: A report of the American College of Cardiology/American Heart Association Task Force on Practice Guidelines (Committee on Management of Acute Myocardial Infarction). Circulation 100(9): 1016-1030.

12. Antman EM, Anbe DT, Armstrong PW, Bates ER, Green LA, et al. (2004) ACC/AHA guidelines for the management of patients with ST-elevation myocardial infarction; A report of the American College of Cardiology/ American Heart Association Task Force on Practice Guidelines (Committee to Revise the 1999 Guidelines for the Management of patients with acute myocardial infarction). J Am Coll Cardiol 44(3): E1211.

13. De Luca G, Suryapranata H, Ottervanger JP, Antman EM (2004) Time delay to treatment and mortality in primary angioplasty for acute myocardial infarction: every minute of delay counts. Circulation 109(10): 1223-1225.

14. Mehta RH, Bufalino VJ, Pan W, Hernandez AF, Cannon CP, et al. (2008) Achieving rapid reperfusion with primary percutaneous coronary intervention remains a challenge: insights from American Heart Association's Get with the Guidelines program. Am Heart J 155(6): 10591067.

15. Bowers TR, O'Neill WW, Grines C, Pica MC, Safian RD, et al. (1998) Effect of reperfusion on biventricular function and survival after right ventricular infarction. N Engl J Med 338(14): 933-940.

16. Schuler G, Hofmann M, Schwarz F, Mehmel H, Manthey J, et al. (1984) Effect of successful thrombolytic therapy on right ventricular function in acute inferior wall myocardial infarction. Am J Cardiol 54(8): 951-957.

17. Raja DC, Chopra A, Subban V, Maharajan R, Anandhan H, et al. (2018) Predictors of short-term outcomes in patients undergoing percutaneous coronary intervention in cardiogenic shock complicating STEMI-A tertiary care center experience. Indian Heart Journal 70(3): S259-64.

18. Robalino BD, Whitlow PL, Underwood DA, Salcedo EE (1989) Electrocardiographic manifestations of right ventricular infarction. Am Heart J 118(1): 138-144.

19. Wellens HJ (1999) The value of the right precordial leads of the electrocardiogram (1999) N Engl J Med 340(5): 381-383. 
20. Anavekar NS, Skali H, Bourgoun M, Ghali JK, Kober L, et al. (2008) Usefulness of right ventricular fractional area change to predict death, heart failure, and stroke following myocardial infarction (from the VALIANT ECHO Study). Am J Cardiol 101(5): 607-612.

21. Zornoff LA, Skali H, Pfeffer MA, St John Sutton M, Rouleau JL, et al. (2002) Right ventricular dysfunction and risk of heart failure and mortality after myocardial infarction. J Am Coll Cardiol 39(9):1450-1455.

\section{ISSN: 2574-1241}

DOI: 10.26717.BJSTR.2019.14.002559

Véronique François. Biomed J Sci \& Tech Res

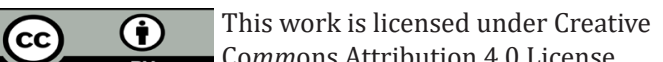

Submission Link: https://biomedres.us/submit-manuscript.php
22. Samad BA, Alam M, Jensen Urstad K (2002) Prognostic impact of right ventricular involvement as assessed by tricuspid annular motion in patients with acute myocardial infarction. Am J Cardiol 90(7): 778-781.

23. Gonzalez Pacheco H, Arias Mendoza A, Alvarez Sangabriel A, Juarez Herrera U, Damas F, et al. (2012) The TIMI risk score for STEMI predicts in-hospital mortality and adverse events in patients without cardiogenic shock undergoing primary angioplasty. Arch Cardiol Mex 82(1): 7-13.

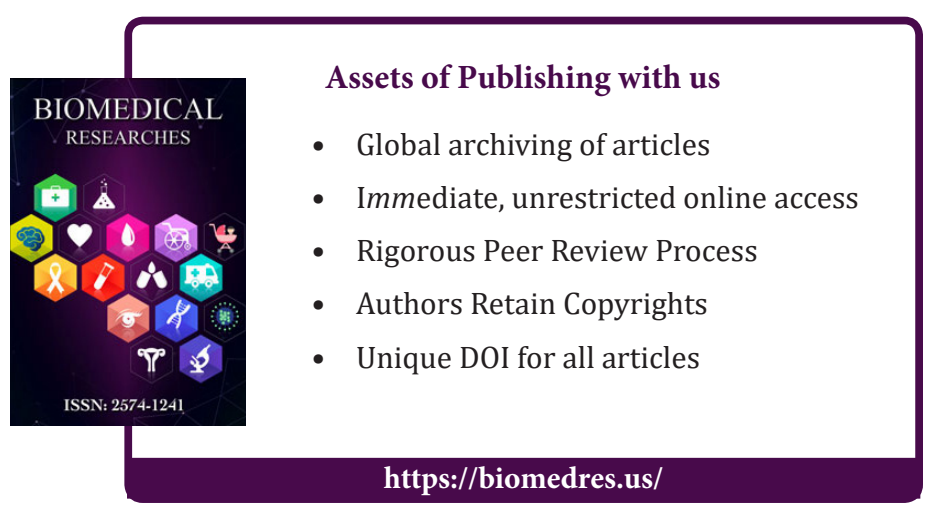

